\title{
AdS Black Hole Solutions in the Extended New Massive Gravity
}

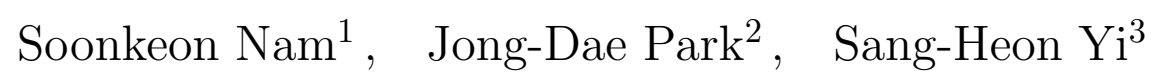 \\ Department of Physics and Research Institute of Basic Science, Kyung Hee University, \\ Seoul 130-701, Korea ${ }^{1,2}$ \\ Department of Physics, College of Sciences, Yonsei University, Seoul 120-749, Korea ${ }^{3}$
}

\begin{abstract}
We have obtained (warped) AdS black hole solutions in the three dimensional extended new massive gravity. We investigate some properties of black holes and obtain central charges of the two dimensional dual CFT. To obtain the central charges, we use the relation between entropy and temperature according to the $A d S / C F T$ dictionary. For $A d S$ black holes, one can also use the central charge function formalism which leads to the same results.
\end{abstract}

\footnotetext{
${ }^{1}$ nam@khu.ac.kr

2jdpark@khu.ac.kr

${ }^{3}$ shyi@yonsei.ac.kr
} 


\section{Introduction}

Recently, there have been some interests in three dimensional gravity theories containing certain higher curvature terms in the action, which are initiated by the advent of the new massive gravity(NMG) 1]. Though there are no propagating degrees of freedom only with the EinsteinHilbert term, the situation becomes different with these higher curvature terms in the Lagrangian. It has long been known that the theory with gravitational Chern-Simons term [2] 3] leads to massive gravitons and also allows black hole solutions [4] $\sim 9]$. The newly proposed NMG theory can be thought as the non-linear extension of Fierz-Pauli massive graviton theory, and it preserves parity symmetry compared to the topologically massive gravity(TMG). Whereas it has also been shown that there are black hole solutions of the usual BTZ type in the NMG with cosmological constants, there are new type of black holes [10] and those with the warped $A d S$ asymptotics [11].

There have been various studies on NMG, which include supersymmetric extension, its black hole solutions, the central charge of the dual conformal field theory (CFT), new type black holes, etc 11 [10] 17]. In the context of the quantum gravity, it is meaningful to consider higher curvature corrections. The NMG may be regarded as three dimensional gravity with curvature square correction terms. Therefore, it is natural to consider even higher curvature corrections in the viewpoint of the quantum gravity or the string theory.

More recently, there are proposals to extend NMG to even higher curvature theories, one of which is the extension of NMG to $R^{3}$ terms in the Lagrangian [18. This is consistent with the holographic c-theorem. The other is the extension to the Born-Infeld type Lagrangian [19]. These may be a good playground to go toward the quantum gravity and to test the $A d S / C F T$ correspondence.

These two extensions become identical up to $R^{3}$ order terms when a suitable matching among parameters is performed. The linearized theory, which tells us the properties of gravitons, is not yet investigated on these theories. Instead of this direction, we investigate the properties of various $A d S$ black holes in these theories and dual conformal field theories.

In this $A d S / C F T$ era, whenever there is a $A d S$ solution in some gravity theory, it is standard to envisage the existence of the dual CFT on the boundary and consider the implications of the gravity for the dual CFT [20] 21] 22]. For the three dimensional gravity theory, it is natural to conjecture the dual theory as a kind of two dimensional CFT. The first thing one may try to do is to obtain the central charge of the hypothetical $2 \mathrm{~d}$ CFT from gravity theory. This is one reason to study the entropy, temperature, etc. in these theories in the view point of $A d S / C F T$ correspondence.

Now, there are several ways to obtain central charges of the dual CFT. In this paper, we use mainly the "Cardy formula" to obtain central charges of the dual CFT 23] 24]. This formula may be thought as the relation between temperature and entropy. Concretely, by writing the black hole entropy in terms of the black hole temperature and interpreting those quantities in the CFT side according to the usual prescription of $A d S_{3} / C F T_{2}$ dictionary, the Cardy formula is given by

$$
S_{B H}=\frac{\pi^{2} L}{3}\left(c_{L} T_{L}+c_{R} T_{R}\right)
$$

Since the extended NMG theories considered in this paper are parity symmetric, the left and the 
right central charge of the dual CFTs are identical $c=c_{L}=c_{R}$. One of the main results in this paper is to present central charge, $c$, of the dual CFTs, explicitly. For the asymptotically AdS case, there is another way to obtain central charge, which is developed in 25] 29, coined as central charge function formalism. We propose a slight extension of this formalism to apply to new type black holes and show that it leads to the value consistent with the above Cardy formula.

This paper is organized as follows. In section 2, we review briefly two types of the extension of NMG proposed recently. One is the extension of NMG with curvature cubic terms $\left(R^{3}-\mathrm{NMG}\right)$ and the other is the Born-Infeld type extension of NMG(BI-NMG). In section 3, after the some explanation of our method to obtain solutions, we present various black hole solutions: BTZ black holes, warped $A d S$ black holes and new type black holes. Various physical quantities, especially the entropy and central charges of dual CFTs are presented. Since this section is relatively long, we divide it into several subsections with some introductory remarks. Our results are summarized and some future directions are discussed in section 4. Some calculational details are relegated to the Appendix.

\section{The Extended New Massive Gravity}

There are new interests in three dimensional gravity theories with higher curvature terms. Though there are no propagating degrees of freedom in three dimensions only with the Einstein-Hilbert term, the situation becomes different with higher curvature terms in the Lagrangian. It has long been known that the theory with gravitational Chern-Simons term [2] [3] leads to massive graviton and also allows black hole solutions. Recently, another type of massive graviton theory has been explored (see for a review [30]), which is named as new massive gravity(NMG).

One reason of these developments comes from the implication of these theories in two dimensional CFT through AdS/CFT correspondence. One may ask whether it is possible to extend NMG theory to the theory with even higher curvature terms. There are recent works to answer this question. In doing so, we need some guideline to go to higher curvature terms. If these theories have a string/M-theory embedding, one can obtain the higher curvature corrections systematically. However, this embedding is not done yet, and moreover, it is unclear that there is such embedding at all. Therefore, it seems desirable to have another way to obtain higher curvature corrections, for instance, through $A d S / C F T$ correspondence. There is a recent attempt [18] to extend the new massive gravity by considering the central charge function and c-theorem, which basically utilize AdS/CFT machinery. There is another attempt [19] containing an infinite number of higher curvature terms, which becomes identical with the previous one up to the curvature cubic order by a suitable parameter matching.

\subsection{The $R^{3}$ extension of New Massive Gravity}

The $R^{3}$ extension of New Massive Gravity $\left(R^{3}-\mathrm{NMG}\right)[18$ is

$$
S=\frac{\eta}{2 \kappa^{2}} \int d^{3} x \sqrt{-g}\left[\sigma R+\frac{2}{l^{2}}+\frac{1}{m^{2}} K+\frac{\xi}{12 \mu^{4}} K^{\prime}\right],
$$


where $2 \kappa^{2}=16 \pi G$ is three dimensional Newton's constant and $\eta, \sigma$ and $\xi$ take 1 or -1 . Here, $K$ and $K^{\prime}$ are defined by

$$
\begin{aligned}
K & =R_{\mu \nu} R^{\mu \nu}-\frac{3}{8} R^{2} \\
K^{\prime} & =17 R^{3}-72 R_{\mu \nu} R^{\mu \nu} R+64 R_{\mu}^{\nu} R_{\nu}^{\rho} R_{\rho}^{\mu} .
\end{aligned}
$$

At the level of equations of motion, the overall sign $\eta$ of the action is meaningless, but it has some important consequence in the black hole entropy and in the positivity of the central charge of dual CFT. So, we allow the overall sign choice $\eta$. Our convention is such that $\kappa^{2}, m^{2}, \mu^{2}$ are always positive, but cosmological constant, $1 / l^{2}$ may be positive or negative.

Note that the above $K$ and $K^{\prime}$ satisfy the following interesting relations

$$
g_{\mu \nu} \frac{\partial K}{\partial R_{\mu \nu}}=-\frac{1}{4} R, \quad g_{\mu \nu} \frac{\partial K^{\prime}}{\partial R_{\mu \nu}}=-24 K .
$$

The equations of motion for the $R^{3}$-NMG are

$$
\sigma G_{\mu \nu}-\frac{1}{l^{2}} g_{\mu \nu}+\frac{1}{2 m^{2}} K_{\mu \nu}-\frac{\xi}{12 \mu^{4}} K_{\mu \nu}^{\prime}=0,
$$

where

$$
\begin{aligned}
K_{\mu \nu}= & g_{\mu \nu}\left(3 R_{\alpha \beta} R^{\alpha \beta}-\frac{13}{8} R^{2}\right)+\frac{9}{2} R R_{\mu \nu}-8 R_{\mu \alpha} R_{\nu}^{\alpha}+\frac{1}{2}\left(4 \nabla^{2} R_{\mu \nu}-\nabla_{\mu} \nabla_{\nu} R-g_{\mu \nu} \nabla^{2} R\right) \\
K_{\mu \nu}^{\prime}= & 17\left[-3 R^{2} R_{\mu \nu}+3 \nabla_{\mu} \nabla_{\nu} R^{2}+\frac{1}{2} g_{\mu \nu} R^{3}-3 g_{\mu \nu} \nabla^{2} R^{2}\right] \\
& -72\left[-2 R R_{\mu \alpha} R_{\nu}^{\alpha}-R_{\alpha \beta} R^{\alpha \beta} R_{\mu \nu}-\nabla^{2}\left(R R_{\mu \nu}\right)+\nabla_{\mu} \nabla_{\nu}\left(R_{\alpha \beta} R^{\alpha \beta}\right)+2 \nabla_{\alpha} \nabla_{(\mu}\left(R_{\nu)}^{\alpha} R\right)\right. \\
& \left.+\frac{1}{2} g_{\mu \nu} R_{\alpha \beta} R^{\alpha \beta} R-g_{\mu \nu} \nabla_{\alpha} \nabla_{\beta}\left(R R^{\alpha \beta}\right)-g_{\mu \nu} \nabla^{2}\left(R_{\alpha \beta} R^{\alpha \beta}\right)\right] \\
& +64\left[-3 R_{\mu}^{\rho} R_{\rho}^{\sigma} R_{\sigma \nu}-\frac{3}{2} \nabla^{2}\left(R_{\mu \alpha} R_{\nu}^{\alpha}\right)+3 \nabla_{\alpha} \nabla_{(\mu}\left(R_{\nu)}^{\beta} R_{\beta}^{\alpha}\right)\right. \\
& \left.+\frac{1}{2} g_{\mu \nu} R_{\alpha \beta} R^{\alpha \beta}-\frac{3}{2} g_{\mu \nu} \nabla_{\alpha} \nabla_{\beta}\left(R_{\rho}^{\alpha} R^{\rho \beta}\right)\right] .
\end{aligned}
$$

\subsection{The Born-Infeld Extension of New Massive Gravity}

The Born-Infeld extension of the new massive gravity (BI-NMG) [19] is also introduced, whose action is

$$
S=-\eta \frac{2 m^{2}}{\kappa^{2}} \int d^{3} x \sqrt{-g}\left[\sqrt{\operatorname{det}\left(\delta^{\mu}{ }_{\nu}+\frac{\sigma}{m^{2}} G^{\mu}{ }^{2}\right)}-1-\frac{1}{2 m^{2} l^{2}}\right],
$$

where $G$ denotes the Einstein tensor, $G^{\mu}{ }_{\nu}=R^{\mu}{ }_{\nu}-\frac{1}{2} \delta^{\mu}{ }_{\nu} R$, and $\eta, \sigma$ will be taken as 1 or -1 .

Expanding the square root expression, one obtains

$$
S=\frac{\eta}{2 \kappa^{2}} \int d^{3} x \sqrt{-g}\left[\sigma R+\frac{2}{l^{2}}+\frac{1}{m^{2}} K-\frac{\sigma}{96 m^{4}} K^{\prime}+\mathcal{O}\left(R^{4}\right)\right]
$$


Note that this covers various cases by taking suitable signs of $\eta$ and $\sigma$. Up to $R^{3}$ terms, the BI action becomes the same form of the previous extended new massive gravity by choosing $\mu^{4}=8 \mathrm{~m}^{4}$ and $\xi=-\sigma$. However, note that the sign of the $R^{3}$ term is fixed in this case in terms of the sign of Einstein-Hilbert term.

Equations of motion for the BI-NMG with $\sigma^{2}=1$ are given by

$$
\begin{aligned}
0= & \sqrt{\operatorname{det} \mathcal{A}}\left[2 \mathcal{B}^{\alpha}{ }_{(\mu} R_{\nu) \alpha}-\mathcal{B} R_{\mu \nu}\right]-2 \sigma m^{2} g_{\mu \nu}\left(\sqrt{\operatorname{det} \mathcal{A}}-1-\frac{1}{2 m^{2} l^{2}}\right) \\
& +g_{\mu \nu}\left[\nabla_{\alpha} \nabla_{\beta}\left(\sqrt{\operatorname{det} \mathcal{A}} \mathcal{B}^{\alpha \beta}\right)-\nabla^{2}(\sqrt{\operatorname{det} \mathcal{A}} \mathcal{B})\right] \\
& +\nabla_{\mu} \nabla_{\nu}(\sqrt{\operatorname{det} \mathcal{A}} \mathcal{B})+\nabla^{2}\left(\sqrt{\operatorname{det} \mathcal{A}} \mathcal{B}_{\mu \nu}\right)-\nabla^{\alpha} \nabla_{\mu}\left(\sqrt{\operatorname{det} \mathcal{A}} \mathcal{B}_{\nu \alpha}\right)-\nabla^{\alpha} \nabla_{\nu}\left(\sqrt{\operatorname{det} \mathcal{A}} \mathcal{B}_{\mu \alpha}\right)
\end{aligned}
$$

where $\mathcal{A}$ and $\mathcal{B}$ are defined by

$$
\mathcal{A}^{\mu}{ }_{\nu} \equiv \delta^{\mu}{ }_{\nu}+\frac{\sigma}{m^{2}} G^{\mu}{ }_{\nu}, \quad \mathcal{B}^{\mu}{ }_{\nu} \equiv\left(\mathcal{A}^{-1}\right)^{\mu}{ }_{\nu}, \quad \mathcal{B} \equiv \mathcal{B}^{\mu}{ }_{\mu} .
$$

\section{Black Hole Solutions}

There are various types of black holes in the above mentioned gravity theories. It has already been known that BTZ black holes are still solutions of any higher curvature gravity theories in three dimensions. Recently, it was shown that there are new type black holes [10] and warped AdS black holes in NMG [11. In this section, we will verify explicitly the same form of the metrics are still solutions in the extended NMG case. Our main results are the verification of the existence of such solutions and central charge expression of the hypothetical dual CFTs.

Since we are dealing with higher curvature theories, the basic tool in obtaining black hole entropy, which we will use, is the so-called Wald formula, pioneered in [32] 34]. This formula was derived by interpreting the black hole entropy as the Noether charge on the horizon. Using simplified version of this formula [35] [36] 37], we will present our results for the entropy of various black holes. By writing the black hole entropy in terms of the black hole temperature and interpreting those quantities in the CFT side according to the usual prescription of $A d S_{3} / C F T_{2}$ correspondence for the parity symmetric case $c=c_{L}=c_{R}$ as

$$
S_{B H}=\frac{\pi^{2} L}{3}\left(c_{L} T_{L}+c_{R} T_{R}\right)=\frac{\pi^{2} L}{3} c\left(T_{L}+T_{R}\right)
$$

we obtain the central charges of the dual CFTs.

According to the $A d S / C F T$ correspondence, the central charges should be written in terms of the parameters in the dual gravity Lagrangian but not of those in black holes, which means that the central charge represents the property of theory but not of a specific state. In other words, when there are different black holes with the same $A d S$ asymptotics, one can expect that the central charges obtained through each black hole system should be the same. In the $R^{3}$ extended NMG case, there are new type black holes of the same asymptotic with BTZ ones, which are, henceforth, expected to lead to the same central charges. In the following, we will show that there are new 
type black holes in the $R^{3}$ extended NMG, like the NMG case, when parameters in the Lagrangian take specific values. Indeed, when parameters are chosen accordingly, the central charge of the dual CFT obtained from these new type black holes becomes identical with the central charge given by BTZ black holes.

We use the dimensional reduction procedure of [11] 31] [38] [40] to find solutions with stationary circular symmetry. This method have already been used to get black hole solutions in TMG and NMG case. According to this method, we can take a metric ansatz with two Killing vectors $\partial_{t}$ and $\partial_{\phi}$ as

$$
d s^{2}=\lambda_{a b}(\rho) d x^{a} d x^{b}+\zeta(\rho)^{-2} R(\rho)^{-2} d \rho^{2},
$$

where $x^{0}=t, x^{1}=\phi, R(\rho)^{2}=-\operatorname{det} \lambda$ and $\zeta(\rho)$ is the scale factor for arbitrary reparametrizations of the radial coordinate $\rho$. The special linear group $S L(2, R)$ in the two Killing vector space is locally isomorphic to the Lorentz group $S O(2,1)$, which suggests the parametrization of the matrix $\lambda$

$$
\lambda=\left(\begin{array}{cc}
T(\rho)+X(\rho) & Y(\rho) \\
Y(\rho) & T(\rho)-X(\rho)
\end{array}\right)
$$

such that special linear transformations of $\lambda$ correspond to Lorentz transformations of the vector $\vec{X}=(T, X, Y)$ and $R^{2}=\vec{X}^{2}=\eta_{i j} X^{i} X^{j}=-T^{2}+X^{2}+Y^{2}$ is the norm in the Minkowski space. As usual, we represent the scalar and vector product of two vectors $\vec{X}$ and $\vec{Y}$ in the following form

$$
\vec{X} \cdot \vec{Y}=\eta_{i j} X^{i} Y^{j}, \quad(\vec{X} \wedge \vec{Y})^{i}=\eta^{i j} \epsilon_{j k l} X^{k} Y^{l},
$$

with $\epsilon_{012}=+1$ for the wedge product.

For the chosen metric ansatz, we can obtain the Ricci tensor components as [1] [9]

$$
\mathcal{R}_{b}^{a}=-\frac{\zeta}{2}\left(\left(\zeta R R^{\prime}\right)^{\prime} \mathbf{1}+(\zeta \ell)^{\prime}\right)_{b}^{a}, \quad \mathcal{R}_{\rho}^{\rho}=-\zeta\left(\zeta R R^{\prime}\right)^{\prime}+\frac{1}{2} \zeta^{2}\left(\vec{X}^{\prime 2}\right), \quad ' \equiv \frac{d}{d \rho},
$$

where $\ell$ represents the matrix defined in terms of the components of the vector $\vec{L}=\left(L^{T}, L^{X}, L^{Y}\right) \equiv$ $\vec{X} \wedge \vec{X}^{\prime}$

$$
\ell=\left(\begin{array}{cc}
-L^{Y} & -L^{T}+L^{X} \\
L^{T}+L^{X} & L^{Y}
\end{array}\right)
$$

In the Appendix we write down the details of this method in our case. Various curvature scalars for the action in the case of $R^{3}$-NMG are given by

$$
\begin{aligned}
\mathcal{R}= & \sigma \zeta^{2}\left\{-2\left(R R^{\prime}\right)^{\prime}+\frac{1}{2}\left(\vec{X}^{\prime 2}\right)\right\}-2 \sigma \zeta \zeta^{\prime} R R^{\prime} \\
K= & \zeta^{4}\left\{\frac{1}{2}\left(\vec{L}^{\prime 2}\right)-\frac{1}{4}\left(R R^{\prime}\right)^{\prime}\left(\vec{X}^{\prime 2}\right)+\frac{5}{32}\left(\vec{X}^{\prime 2}\right)^{2}\right\} \\
& +\zeta \zeta^{\prime}\left\{\left(\vec{L} \cdot \vec{L}^{\prime}\right)-\frac{1}{4} R R^{\prime}\left(\vec{X}^{\prime 2}\right)\right\}+\frac{1}{2} \zeta^{2} \zeta^{\prime 2}\left(\vec{L}^{2}\right) \\
K^{\prime}= & \zeta^{6}\left\{-\frac{3}{2}\left(R R^{\prime}\right)^{\prime}\left(\vec{X}^{\prime 2}\right)^{2}+\frac{9}{8}\left(\vec{X}^{\prime 2}\right)^{3}+24\left(R R^{\prime}\right)^{\prime}\left(\vec{L}^{\prime 2}\right)-18\left(\vec{X}^{\prime 2}\right)\left(\vec{L}^{\prime 2}\right)\right\}
\end{aligned}
$$




$$
\begin{aligned}
& +\zeta^{5} \zeta^{\prime}\left\{-\frac{3}{2}\left(R R^{\prime}\right)^{\prime}\left(\vec{X}^{\prime 2}\right)^{2}+24\left(R R^{\prime}\right)\left(\vec{L}^{\prime 2}\right)+48\left(R R^{\prime}\right)^{\prime}\left(\vec{L} \cdot \vec{L}^{\prime}\right)\right. \\
& \left.-36\left(\vec{X}^{\prime 2}\right)\left(\vec{L} \cdot \vec{L}^{\prime}\right)\right\}+\zeta^{4} \zeta^{\prime 2}\left\{24\left(R R^{\prime}\right)^{\prime}\left(\vec{L}^{2}\right)-18\left(\vec{X}^{\prime 2}\right)\left(\vec{L} \cdot \vec{L}^{\prime}\right)\right\} \\
& +24 \zeta^{3} \zeta^{\prime 3}\left(R R^{\prime}\right)\left(\vec{L}^{2}\right) .
\end{aligned}
$$

Using these expressions, one can reduce the $R^{3}$-NMG action to the one-dimensional form given in the Appendix up to a surface term. From the reduced action, we can derive the reduced equations of motion. After variation of $\zeta$ we can fix the gauge $\zeta=$ const using the reparametrization invariance of the metric (17). The first form (A.1) of the reduced action in the Appendix gives us the equation of motion after variation with respect to $\vec{X}$ as

$$
\delta A+\frac{1}{\zeta^{2}} \delta E+\frac{1}{\zeta^{4}} \delta H=0
$$

where $\delta A, \delta E$ and $\delta H$ are written down explicitly in Eq. (A.4) (A.6) in the Appendix. It is convenient to use the second form (A.2) of the reduced action for the variation with respect to $\zeta$, which gives us the following Hamiltonian constraint :

$$
\mathcal{H} \equiv\left(5 A-B^{\prime}\right)+\frac{1}{\zeta^{2}}\left(3 E-F^{\prime}\right)+\frac{1}{\zeta^{4}}\left(H-J^{\prime}\right)-\frac{2}{\ell^{2}} \frac{1}{\zeta^{6}}=0 .
$$

In the BI-NMG case, we can obtain the equation of motion through the variation of the action with respect to $\vec{X}$

$$
\begin{array}{r}
0=4(\operatorname{det} \mathcal{A})^{2}\left(\vec{S}^{\prime \prime}-\vec{Q}^{\prime}+\vec{P}\right)-4(\operatorname{det} \mathcal{A})\left(\operatorname{det} \mathcal{A}^{\prime}\right) \vec{S}^{\prime}-2(\operatorname{det} \mathcal{A})\left(\operatorname{det} \mathcal{A}^{\prime \prime}\right) \vec{S} \\
+3\left(\operatorname{det} \mathcal{A}^{\prime}\right)^{2} \vec{S}+2(\operatorname{det} \mathcal{A})\left(\operatorname{det} \mathcal{A}^{\prime}\right) \vec{Q}
\end{array}
$$

and from the variation with respect to $\zeta$, we can also obtain the Hamiltonian constraint,

$$
0=\frac{1}{2} \frac{\left(\operatorname{det} \mathcal{A}^{\prime}\right)}{\operatorname{det} \mathcal{A}} \mathcal{N}+\mathcal{M}-\mathcal{N}^{\prime}-2(\operatorname{det} \mathcal{A})+2(\operatorname{det} \mathcal{A})^{1 / 2}\left(1+\frac{1}{2 m^{2} \ell^{2}}\right),
$$

where $\mathcal{M}, \mathcal{N}$ and $\operatorname{det} \mathcal{A}$ are written down explicitly in Eq. A.23, (A.24) and (A.12) in the Appendix. The variation of the reduced action in both $R^{3}$-NMG and BI-NMG cases are presented in detail in the Appendix.

In order to obtain exact solutions, we can take a vector $\vec{X}$ as follows :

$$
\vec{X}=\vec{\alpha} \rho^{2}+\vec{\beta} \rho+\vec{\gamma}
$$

where $\vec{\alpha}, \vec{\beta}$ and $\vec{\gamma}$ are linearly independent constant vectors. Substituting this ansatz into equation (15), we have to impose the following constraints on vectors $\vec{\alpha}$ and $\vec{\beta}$ in order to vanish the higher than the third order components which is given by

$$
\vec{\alpha}^{2}=0, \quad(\vec{\alpha} \cdot \vec{\beta})=0 .
$$

These constraints induce two more constraints

$$
\vec{\alpha} \wedge \vec{\beta}=b \vec{\alpha}, \quad \vec{\beta}^{2}=b^{2},
$$


with a real constant $b$. Using these conditions with $(\vec{\alpha} \cdot \vec{\gamma}) \equiv-z$, we can obtain the equation of motion and the Hamiltonian constraint in the $R^{3}$-NMG case as

$$
\begin{aligned}
& 0=\left[\xi\left(-\frac{33}{8} b^{4}+30 b^{2} z+24 z^{2}\right)-\frac{\mu^{4}}{m^{2} \zeta^{2}}\left(\frac{17}{4} b^{2}+2 z\right)-2 \frac{\mu^{4}}{\zeta^{4}} \sigma\right] \vec{\alpha}, \\
& 0=\frac{\xi}{12 \mu^{4}}\left\{-\frac{3}{8} b^{6}-216 b^{2} z^{2}+36 b^{4} z-384 z^{3}\right\}+\frac{1}{\zeta^{2} m^{2}}\left\{2 z^{2}-\frac{1}{32} b^{4}+3 b^{2} z\right\}+\frac{\sigma}{\zeta^{4}} \frac{b^{2}}{2}-\frac{2}{\ell^{2} \zeta^{6}} .
\end{aligned}
$$

In the BI-NMG case, we obtain

$$
\operatorname{det} \mathcal{A}=\left(1+\sigma \frac{\zeta^{2}}{m^{2}} \frac{b^{2}}{4}\right)^{2}\left\{1+\sigma \frac{\zeta^{2}}{m^{2}}\left(\frac{b^{2}}{4}-2 z\right)\right\}
$$

and note that $\operatorname{det} \mathcal{A}^{\prime}=\operatorname{det} \mathcal{A}^{\prime \prime}=0$ under the above conditions. The equation of motion and the Hamiltonian constraint are given by

$$
\begin{aligned}
& 0=\left[\sigma \frac{\zeta^{2}}{m^{2}}+\frac{5}{2} \frac{\zeta^{4}}{m^{4}} b^{2}+\sigma \frac{9}{16} \frac{\zeta^{6}}{m^{6}} b^{4}\right] \vec{\alpha} \\
& 0=1+\sigma \frac{\zeta^{2}}{m^{2}}\left(\frac{1}{4} b^{2}-z\right)+\frac{\zeta^{4}}{m^{4}}\left(\frac{3}{4} b^{2} z\right)-\left(1+\frac{1}{2 m^{2} \ell^{2}}\right)\left[1+\sigma \frac{\zeta^{2}}{m^{2}}\left(\frac{1}{4} b^{2}-2 z\right)\right]^{1 / 2}
\end{aligned}
$$

As is given in the following, the case of $\vec{\alpha}=0$ in the Eq.s (22) and (25) leads to BTZ black holes, whereas the other condition of $\vec{\alpha} \neq 0$ leads to warped black hole solutions.

This section is organized as follows. BTZ black holes are presented in subsection 3.1 with some explanation of generic features. In subsection 3.2, we present warped black hole solutions. Finally, in subsection 3.3, we present new type black holes and propose some straightforward extension of central charge function formalism for applying it to new type black holes.

\subsection{BTZ black holes}

If we consider the condition $\vec{\alpha}=0$, then the vector ansatz (19) reduces to

$$
\vec{X}=\vec{\beta} \rho+\vec{\gamma} .
$$

Taking two vectors $\vec{\beta}$ and $\vec{\gamma}$ to be $\vec{\beta}=\left[-\left(1-\ell^{2}\right) / \ell^{2},-\left(1+\ell^{2}\right) / \ell^{2}, 0\right]$ and $\vec{\gamma}=\left[\tilde{m}\left(1+\ell^{2}\right) / 4, \tilde{m}(1-\right.$ $\left.\ell^{2}\right) / 4,-j / 2$ ], then we find that the metric ansatz (7) becomes the BTZ black hole solution [41] 42]

$$
d s^{2}=\left(-\frac{2}{\ell^{2}} \rho+\frac{\tilde{m}}{2}\right) d t^{2}-j d t d \phi+\left(2 \rho+\frac{\tilde{m} \ell^{2}}{2}\right) d \phi^{2}+\frac{d \rho^{2}}{\left(\frac{4}{\ell^{2}} \rho^{2}-\frac{\tilde{m}^{2} \ell^{2}-j^{2}}{4}\right)},
$$

for $\zeta=1$ and $b^{2}=4 / \ell^{2}$ [11]. If we change the coordinate $\rho=r^{2} / 2-\tilde{m} \ell^{2} / 4$, then we can transform the $(t, \rho, \phi)$ coordinate system into the $(t, r, \phi)$ one. We can also reparametrize $r \rightarrow \ell r, t \rightarrow-L t$ 
and $\phi \rightarrow L \phi / \ell$ in order for $t$ and $r$ to be dimensionless. With $\zeta=\ell / L$ and $b^{2}=4 / \ell^{2}$ we can see that BTZ black holes are solutions of the above equations of motion, of which metric is given by

$$
d s^{2}=L^{2}\left[-\frac{\left(r^{2}-r_{+}^{2}\right)\left(r^{2}-r_{-}^{2}\right)}{r^{2}} d t^{2}+\frac{r^{2}}{\left(r^{2}-r_{+}^{2}\right)\left(r^{2}-r_{-}^{2}\right)} d r^{2}+r^{2}\left(d \phi+\frac{r_{+} r_{-}}{r^{2}} d t\right)^{2}\right],
$$

where $L$ is the $A d S$ length. $L$ should always be positive and it is natural to write physical quantities in terms of this $A d S$ length, $L$.

The Hawking temperature of the black hole can be obtained by dividing $2 \pi$ of the surface gravity $\kappa$, which is, for the above ADM form of the metric, given by

$$
\kappa=\left.\left(-\frac{1}{2} \nabla_{\mu} \xi_{\nu} \nabla^{\mu} \xi^{\nu}\right)^{1 / 2}\right|_{r=r_{+}}=\left.\frac{1}{L} \frac{\partial_{r} N}{\sqrt{g_{r r}}}\right|_{r=r_{+}}
$$

where $\xi$ is a null Killing vector at the Horizon with the normalization as $\xi^{2} \rightarrow-r^{2}$ for $r \rightarrow \infty$. This gives us

$$
T_{H}=\frac{r_{+}}{2 \pi L}\left(1-\frac{r_{-}^{2}}{r_{+}^{2}}\right) .
$$

Another way to obtain the Hawking temperature is to demand the regularity of the Euclideanized form of the black hole metric with an appropriate choice of time coordinate scale. The regularity condition in this case leads to the periodicity of the Euclidean time and then it can be interpreted as the temperature. Using this approach, one obtains the same result with the above one in our case. The angular velocity at the horizon can be defined as

$$
\Omega_{H}=\frac{1}{L} N^{\phi}\left(r_{+}\right)=\frac{1}{L} \frac{r_{-}}{r_{+}} .
$$

For BTZ black holes, the left and the right temperatures are given by [43]

$$
T_{L}=\frac{r_{+}+r_{-}}{2 \pi L}, \quad T_{R}=\frac{r_{+}-r_{-}}{2 \pi L} .
$$

For higher curvature gravity theories, there are some difficulties to define conserved charges and we need new ingredients to define various physical quantities like mass and angular momentum [44]. Strictly speaking, it is required to define various physical quantities in concrete ways and verify the first law of black holes systematically. However, we will bypass these by assuming the validity of the first law of black holes in these higher curvature gravity theories. Then, the mass and angular momentum of these black holes may be read by integrating the first law of black hole thermodynamics

$$
d M=T_{H} d S_{B H}+\Omega_{H} d J
$$

Note that the left and the right energies can also be defined as

$$
E_{L} \equiv \frac{\pi^{2} L}{6} c_{L} T_{L}^{2}, \quad E_{R} \equiv \frac{\pi^{2} L}{6} c_{R} T_{R}^{2},
$$

which are related to mass and angular momentum in the BTZ case as

$$
M=E_{L}+E_{R}, \quad J=L\left(E_{L}-E_{R}\right) .
$$


For the parity symmetric case, one can see, through the above relations, that the mass and angular momentum are proportional to the central charge $c=c_{L}=c_{R}$ of dual CFT, and check in both cases that the integral Smarr relation holds as

$$
M=\frac{1}{2} T_{H} S_{B H}+\Omega_{H} J
$$

\section{$\underline{R^{3} \text {-NMG case }}$}

The condition $\vec{\alpha}=0$ gives the BTZ black hole solution (29), then this solution has to satisfy the condition (23). With $b^{2}=4 / l^{2}$ and $\zeta=l / L, L^{2}$ should satisfy

$$
\sigma-\frac{L^{2}}{l^{2}}-\frac{1}{4 m^{2} L^{2}}-\frac{\xi}{\mu^{4} L^{4}}=0 \text {. }
$$

The entropy is

$$
S_{B H}=\frac{A_{H}}{4 G} \eta\left(\sigma+\frac{1}{2 m^{2} L^{2}}+\frac{\xi}{\mu^{4} L^{4}}\right), \quad A_{H} \equiv 2 \pi L r_{+},
$$

where $L^{2}$ is given by the solution of Eq. (33). The mass and angular momentum are given by

$$
M=\frac{r_{+}^{2}+r_{-}^{2}}{8 G} \eta\left(\sigma+\frac{1}{2 m^{2} L^{2}}+\frac{\xi}{\mu^{4} L^{4}}\right), \quad J=\frac{L r_{+} r_{-}}{4 G} \eta\left(\sigma+\frac{1}{2 m^{2} L^{2}}+\frac{\xi}{\mu^{4} L^{4}}\right) .
$$

From Cardy formula, one obtains the central charge for of the dual $2 \mathrm{~d}$ CFT as

$$
c=\frac{3 L}{2 G} \eta\left[\sigma+\frac{1}{2 m^{2} L^{2}}+\frac{\xi}{\mu^{4} L^{4}}\right] .
$$

Note that the above mass and angular momentum are proportional to the central charge, indeed.

For a comparison, let us consider the central charge of the dual CFT obtained from the NMG $A d S$ black hole solution written in the same form with (36). In our convention, 1 , it is given by

$$
c=\frac{3 L}{2 G} \eta\left[\sigma+\frac{1}{2 m^{2} L^{2}}\right]
$$

where

$$
L^{2} \equiv \frac{l^{2}}{2}\left[\sigma+\sqrt{1-\frac{1}{m^{2} l^{2}}}\right]
$$

One can see that $\eta=\sigma=1$ is the sign choice in [10] and $\eta=\sigma=-1$ is the choice in [11].

\section{BI-NMG case}

In this case we have to consider the constraint (26) with $b^{2}=4 / l^{2}$ and $\zeta=l / L$, then $L^{2}$ should satisfy

$$
\frac{1}{L^{2}}=\frac{\sigma}{l^{2}}\left(1+\frac{1}{4 m^{2} l^{2}}\right)
$$

where $1 / l^{2}$ is cosmological constant and may be negative, whereas $A d S$ length, $L$, should be always positive. One of interesting points of the higher curvature theories is that there are asymptotically $A d S$ solutions even though positive cosmological constants.

\footnotetext{
${ }^{1}$ This is consistent with results in $[12 \sim[10]$.
} 
The entropy in this case is given by

$$
\begin{aligned}
S_{B H} & =-\left.\eta \sigma \frac{A_{H}}{4 G} \sqrt{\operatorname{det} \mathcal{A}}\left(\mathcal{B}^{t t}\left(g^{t t}\right)^{-1}+\mathcal{B}^{r r}\left(g^{r r}\right)^{-1}-\mathcal{B}\right)\right|_{r=r_{+}} \\
& =\eta \sigma \frac{A_{H}}{4 G} \sqrt{1+\sigma \frac{1}{m^{2} L^{2}}}=\eta \sigma \frac{\pi L r_{+}}{2 G} \sqrt{1+\sigma \frac{1}{m^{2} L^{2}}} .
\end{aligned}
$$

By substituting $L$ in terms of $l$ and $m$, the entropy may be written in terms of parameters in the Lagrangian with $r_{+}$, for instance $\eta=\sigma=1$, as

$$
S_{B H}=\frac{\pi l r_{+}}{2 G}\left(1+\frac{1}{2 m^{2} l^{2}}\right)\left(1+\frac{1}{4 m^{2} l^{2}}\right)^{-1 / 2} .
$$

The mass of these black holes are given by

$$
M=\eta \sigma \frac{r_{+}^{2}+r_{-}^{2}}{8 G} \sqrt{1+\sigma \frac{1}{m^{2} L^{2}}}, \quad J=\eta \sigma \frac{L r_{+} r_{-}}{4 G} \sqrt{1+\sigma \frac{1}{m^{2} L^{2}}} .
$$

Noting that the Born-Infeld Lagrangian is also parity-symmetric, one can obtain the central charge $c=c_{L}=c_{R}$ of the dual $2 \mathrm{~d}$ CFT as

$$
c=\eta \sigma \frac{3 L}{2 G} \sqrt{1+\sigma \frac{1}{m^{2} L^{2}}} .
$$

Note that the positive central charge is allowed only when $\eta \sigma=1$, which is the standard sign choice of the Einstein-Hilbert term.

All the above central charges for BTZ black holes can also be obtained by the central charge function formalism, which may be thought as the compact summary of the above procedure:

$$
c \equiv \frac{L}{2 G} g_{\mu \nu} \frac{\partial \mathcal{L}}{\partial R_{\mu \nu}} .
$$

For instance, in the BI case

$$
c=\eta \sigma \frac{L}{2 G} \sqrt{\operatorname{det} \mathcal{A}} \mathcal{B}
$$

where

$$
\sqrt{\operatorname{det} \mathcal{A}}=\left(1+\sigma \frac{1}{m^{2} L^{2}}\right)^{3 / 2}, \quad \mathcal{B}=3\left(1+\sigma \frac{1}{m^{2} L^{2}}\right)^{-1}
$$

\subsection{Warped AdS Solutions}

Setting the condition $\vec{\beta}^{2}=b^{2}=1,(\vec{\beta} \cdot \vec{\gamma})=0, \vec{\gamma}^{2}=-\beta^{2} \rho_{0}^{2}$ and $(1-2 z)=\beta^{2}$, then we can get $R^{2}=(1-2 z) \rho^{2}+\vec{\gamma}^{2}=\beta^{2}\left(\rho^{2}-\rho_{0}^{2}\right)$. In addition to this condition we can choose vectors as follows [1]

$$
\begin{aligned}
& \vec{\alpha}=(1 / 2,-1 / 2,0), \quad \vec{\beta}=(\omega,-\omega,-1), \\
& \vec{\gamma}=(z+u, z-u,-2 \omega z) \quad\left(u=\beta^{2} \rho_{0}^{2} / 4 z+\omega^{2} z\right),
\end{aligned}
$$

which lead to the metric form

$$
d s^{2}=-\frac{\beta^{2}\left(\rho^{2}-\rho_{0}^{2}\right)}{\Delta^{2}} d t^{2}+\frac{d \rho^{2}}{\zeta^{2} \beta^{2}\left(\rho^{2}-\rho_{0}^{2}\right)}+\Delta^{2}\left(d \phi-\frac{\rho+\left(1-\beta^{2}\right) \omega}{\Delta^{2}} d t\right)^{2},
$$


where $\Delta^{2}$ is defined by

$$
\Delta^{2}=\rho^{2}+2 \omega \rho+2 u=\rho^{2}+2 \omega \rho+\left(1-\beta^{2}\right) \omega^{2}+\frac{\beta^{2} \rho_{0}^{2}}{1-\beta^{2}} .
$$

If we take $\zeta^{2}=3 /\left(4 \beta^{2}-1\right) \cdot 4 / L^{2}$ and reparametrize $t \rightarrow t /\left(\zeta \beta^{2}\right)$ and $\phi \rightarrow \phi / \zeta$, then we can obtain warped AdS black hole solutions as follows:

$$
d s^{2}=\frac{4 \beta^{2}-1}{12 \beta^{2}} L^{2}\left[-\frac{\rho^{2}-\rho_{0}^{2}}{\Delta^{2}} d t^{2}+\frac{d \rho^{2}}{\rho^{2}-\rho_{0}^{2}}+\beta^{2} \Delta^{2}\left(d \phi-\frac{\rho+\left(1-\beta^{2}\right) \omega}{\beta^{2} \Delta^{2}} d t\right)^{2}\right],
$$

where we have chosen the overall coefficient such that $L$ corresponds to the Ricci scalar curvature as $R=-\frac{6}{L^{2}}$. To see this, one may note that the asymptotic region is given by taking $\rho \rightarrow \infty$ as

$$
d s^{2} \simeq \frac{4 \beta^{2}-1}{12 \beta^{2}} L^{2}\left[-d t^{2}+\frac{d \rho^{2}}{\rho^{2}}+\beta^{2} \rho^{2}\left(d \phi-\frac{1}{\beta^{2}} \frac{1}{\rho} d t\right)^{2}\right]
$$

which can be shown to be the warped $A d S_{3}$ in Poincaré coordinates by a suitable change of variables 2 It is an interesting fact that the above warped $A d S_{3}$ black hole metric has constant curvature invariants of the same values with the warped $A d S_{3}$, which allows to obtain it by the quotienting method from the warped $A d S_{3}$ space [29] [45].

By a suitable coordinate transformation with parameters relations

$$
\rho_{0}=\frac{1}{2}\left(r_{+}-r_{-}\right), \quad\left(1-\beta^{2}\right) \omega=\frac{1}{2}\left(r_{+}+r_{-}-2 \beta \sqrt{r_{+} r_{-}}\right), \quad \beta^{2}=\frac{\nu^{2}+3}{4 \nu^{2}},
$$

the above metric can be written as [45]

$$
d s^{2}=L^{2}\left[-N(r)^{2} d t^{2}+R(r)^{2}\left(d \theta+N^{\theta}(r) d t\right)^{2}+\frac{d r^{2}}{4 R(r)^{2} N(r)^{2}}\right]
$$

where

$$
\begin{aligned}
N(r)^{2} & =\frac{\left(\nu^{2}+3\right)\left(r-r_{+}\right)\left(r-r_{-}\right)}{4 R(r)^{2}} \\
N^{\theta}(r) & =\frac{2 \nu r-\sqrt{r_{+} r_{-}\left(\nu^{2}+3\right)}}{2 R(r)^{2}} \\
R(r)^{2} & =\frac{r}{4}\left(3\left(\nu^{2}-1\right) r+\left(\nu^{2}+3\right)\left(r_{+}+r_{-}\right)-4 \nu \sqrt{r_{+} r_{-}\left(\nu^{2}+3\right)}\right) .
\end{aligned}
$$

We will use this form of the metric for the entropy, Hawking temperature, etc in the following.

The black hole temperature and the angular velocity are given by

$$
T_{H}=\frac{\beta^{2}}{2 \pi L} \sqrt{\frac{3}{4 \beta^{2}-1}}\left(\frac{r_{+}-r_{-}}{r_{+}-\beta \sqrt{r_{+} r_{-}}}\right), \quad \Omega_{H}=\frac{1}{L} \sqrt{\frac{4 \beta^{2}-1}{3}}\left(\frac{1}{r_{+}-\beta \sqrt{r_{+} r_{-}}}\right) .
$$

\footnotetext{
${ }^{2}$ The coordinate transformations are not unique at the asymptotic region. For coordinate transformation to the Schwarzschild type, see [4].
} 
The left and the right temperatures of these warped $A d S$ black holes are given by [45]

$$
T_{L}=\frac{r_{+}+r_{-}-2 \beta \sqrt{r_{+} r_{-}}}{2 \pi L}\left(\frac{3 \beta^{2}}{4 \beta^{2}-1}\right), \quad T_{R}=\frac{r_{+}-r_{-}}{2 \pi L}\left(\frac{3 \beta^{2}}{4 \beta^{2}-1}\right),
$$

which are related to the above Hawking temperature and angular velocity as

$$
\frac{1}{T_{H}}=\pi L \sqrt{\frac{4 \beta^{2}-1}{3 \beta^{4}}}\left(\frac{T_{L}+T_{R}}{T_{R}}\right), \quad \frac{\Omega_{H}}{T_{H}}=\frac{1}{T_{R} L} .
$$

It was suggested in [45] that more useful charges in the warped case are the left and the right moving energies which are defined by

$$
E_{L} \equiv \frac{\pi^{2} L}{6} c_{L} T_{L}^{2}, \quad E_{R} \equiv \frac{\pi^{2} L}{6} c_{R} T_{R}^{2}
$$

By construction, these charges satisfy

$$
\frac{\partial S_{B H}}{\partial E_{L}}=\frac{1}{T_{L}}, \quad \frac{\partial S_{B H}}{\partial E_{R}}=\frac{1}{T_{R}} .
$$

The mass and angular momentum can be related to these charges a 3

$$
M=\sqrt{\frac{3 \beta^{4}}{4 \beta^{2}-1}} \sqrt{\frac{2 c_{L} E_{L}}{3 L}}, \quad J=L\left(E_{L}-E_{R}\right) .
$$

One can check that $(M, J)$ defined in the above satisfy, through the formula (49), the first law of black holes,

$$
d M=T_{H} d S_{B H}+\Omega_{H} d J .
$$

By using the left and the right temperatures in terms of black hole parameters $r_{+}, r_{-}$and recalling $c_{L}=c_{R}=c$ in our case, one may write the mass and the angular momentum in terms of the central charges as follows:

$$
\begin{aligned}
M & =\frac{\beta}{6 L}\left(\frac{3 \beta^{2}}{4 \beta^{2}-1}\right)^{3 / 2}\left[r_{+}+r_{-}-2 \beta \sqrt{r_{+} r_{-}}\right] c \\
J & =\frac{1}{24}\left(\frac{3 \beta^{2}}{4 \beta^{2}-1}\right)^{2}\left[\left(r_{+}+r_{-}-2 \beta \sqrt{r_{+} r_{-}}\right)^{2}-\left(r_{+}-r_{-}\right)^{2}\right] c
\end{aligned}
$$

where $c$ denotes the central charge of dual CFT. One may also write $(M, J)$ in terms of the black entropy since the central charges are proportional to the entropy in the present case.

Using black hole entropy, $S_{B H}$, given in the following, one can show that the above formula satisfies Smarr-like relation

$$
M=T_{H} S_{B H}+2 \Omega_{H} J,
$$

and verify the differential form of the first law of black holes explicitly. We will not present the mass and angular momentum explicitly in each of the extended NMG's, since it is straightforward to obtain those for the given central charges which are given in the following.

\footnotetext{
${ }^{3}$ We have modified slightly the relations given in 45] in order to apply these to our case. These modified ones also lead to the correct results in TMG case.
} 
$\underline{R^{3}-\mathrm{NMG} \text { case }}$

Substituting the values $b^{2}=1, z=\left(1-\beta^{2}\right) / 2$ and $\zeta^{2}=3 /\left(4 \beta^{2}-1\right) \cdot 4 / L^{2}$ into two equations (22) and (23), then we can see that $L$ and $\beta$ should satisfy two equations

$$
\begin{aligned}
0 & =\sigma-\frac{3\left(4 \beta^{2}-21\right)}{2\left(4 \beta^{2}-1\right)} \frac{1}{m^{2} L^{2}}-\xi \frac{27\left(4 \beta^{2}-3\right)\left(4 \beta^{2}-15\right)}{\left(4 \beta^{4}-1\right)^{2}} \frac{1}{\mu^{4} L^{4}}, \\
0 & =\sigma-\frac{4 \beta^{2}-1}{3} \frac{L^{2}}{l^{2}}+\frac{3\left(16 \beta^{4}-80 \beta^{2}+63\right)}{4 \beta^{2}-1} \frac{1}{4 m^{2} L^{2}}+\frac{9\left(4 \beta^{2}-3\right)\left(32 \beta^{4}-108 \beta^{2}+75\right)}{\left(4 \beta^{4}-1\right)^{2}} \frac{\xi}{\mu^{4} L^{4}} .
\end{aligned}
$$

Note that $L$ can be solved as

$$
L^{2}=\frac{\sigma}{\left(4 \beta^{2}-1\right)} \frac{3}{4 m^{2} \mu^{2}}\left\{\left(4 \beta^{2}-21\right) \mu^{2} \pm \sqrt{\left(4 \beta^{2}-21\right)^{2} \mu^{4}+48 \xi \sigma\left(4 \beta^{2}-3\right)\left(4 \beta^{2}-15\right) m^{4}}\right\} .
$$

Using Wald's formula, one obtains

$$
\begin{aligned}
S_{B H} & =\frac{A_{H}}{4 G} \eta\left[\sigma+\frac{3\left(5-4 \beta^{2}\right)}{2\left(4 \beta^{2}-1\right) m^{2} L^{2}}-\xi \frac{9\left(3-4 \beta^{2}\right)\left(13-12 \beta^{2}\right)}{\left(4 \beta^{4}-1\right)^{2} \mu^{4} L^{4}}\right] \\
A_{H} & \equiv 2 \pi L R\left(r_{+}\right)=2 \pi L \sqrt{\frac{3}{4 \beta^{2}-1}}\left(r_{+}-\beta \sqrt{r_{+} r_{-}}\right)
\end{aligned}
$$

where $\beta$ and $L^{2}$ are given by the solutions of the above equation.

The central charges for the warped $A d S$ black holes can also be obtained by the Cardy formula

$$
S=\frac{\pi^{2} L}{3}\left(c_{L} T_{L}+c_{R} T_{R}\right)=\frac{\pi^{2} L}{3} c\left(T_{L}+T_{R}\right),
$$

which leads to

$$
c=\frac{L}{2 G} \frac{\sqrt{3\left(4 \beta^{2}-1\right)}}{\beta^{2}} \eta\left[\sigma+\frac{3\left(5-4 \beta^{2}\right)}{2\left(4 \beta^{2}-1\right) m^{2} L^{2}}-\xi \frac{9\left(3-4 \beta^{2}\right)\left(13-12 \beta^{2}\right)}{\left(4 \beta^{4}-1\right)^{2} \mu^{4} L^{4}}\right] .
$$

The naive application of the central charge function formalism leads to

$$
c=\frac{3 L}{2 G} \eta\left[\sigma+\frac{1}{2 m^{2} L^{2}}-\xi \frac{3\left(4 \beta^{2}-3\right)\left(4 \beta^{2}-7\right)}{\left(4 \beta^{4}-1\right)^{2} \mu^{4} L^{4}}\right],
$$

which gives the different answer in this case, which is not unexpected since the central charge function formalism is developed for the $A d S$ space not warped $A d S$ space.

One can see that the above result (54) reproduces the new massive gravity case if one set $\xi=0$

$$
\begin{aligned}
\beta^{2} & =\frac{1}{4\left(1-m^{2} l^{2}\right)}\left(21-9 m^{2} l^{2} \pm 2 \sqrt{3\left(7+5 m^{2} l^{2}\right) m^{2} l^{2}}\right) \\
\frac{1}{L^{2}} & =\frac{2\left(4 \beta^{2}-1\right) \sigma m^{2}}{3\left(4 \beta^{2}-21\right)}=\frac{2 \sigma}{63 l^{2}}\left(-39 m^{2} l^{2} \pm 10 \sqrt{3\left(7+5 m^{2} l^{2}\right) m^{2} l^{2}}\right)
\end{aligned}
$$

which implies

$$
S_{B H}=\frac{A_{H}}{4 G} \eta\left[\sigma+\frac{3\left(5-4 \beta^{2}\right)}{2\left(4 \beta^{2}-1\right) m^{2} L^{2}}\right]=\frac{A_{H}}{4 G} \frac{16 \eta \sigma}{21-4 \beta^{2}}=\frac{A_{H}}{4 G} \eta\left(\frac{4}{5} \sigma-\frac{6}{5 m^{2} L^{2}}\right),
$$


and

$$
c=\eta \sigma \frac{8 L}{G} \frac{\sqrt{3\left(4 \beta^{2}-1\right)}}{\beta^{2}\left(21-4 \beta^{2}\right)} .
$$

These results are consistent with the choice of $\eta=\sigma=-1$ in [46].

$\underline{\text { BI-NMG case }}$

Substituting the values $b^{2}=1, z=\left(1-\beta^{2}\right) / 2$ and $\zeta^{2}=3 /\left(4 \beta^{2}-1\right) \cdot 4 / L^{2}$ into the equations (25) and (26), we obtain

$$
\begin{aligned}
L^{2} & =-\frac{27 \sigma}{\left(4 \beta^{2}-1\right) m^{2}}, \\
0 & =1+\frac{3\left(2 \beta^{2}-1\right)}{4 \beta^{2}-1} \frac{\sigma}{m^{2} L^{2}}-\frac{54\left(\beta^{2}-1\right)}{\left(4 \beta^{2}-1\right)^{2}} \frac{1}{m^{4} L^{4}}-\left(1+\frac{1}{2 m^{2} l^{2}}\right)\left[1+\frac{3\left(4 \beta^{2}-3\right)}{4 \beta^{2}-1} \frac{\sigma}{m^{2} L^{2}}\right]^{1 / 2},
\end{aligned}
$$

where we may note that there are warped solutions only for $\sigma=-1$.

Using Wald's formula, one obtains $\left(A_{H} \equiv 2 \pi L R\left(r_{+}\right)\right)$

$$
\begin{aligned}
S_{B H} & =-\left.\eta \sigma \frac{A_{H}}{4 G} \sqrt{\operatorname{det} \mathcal{A}}\left(\mathcal{B}^{t t}\left(g^{t t}\right)^{-1}+\mathcal{B}^{r r}\left(g^{r r}\right)^{-1}-\mathcal{B}\right)\right|_{\rho=\rho_{0}}, \\
& =\eta \sigma \frac{A_{H}}{4 G}\left(1+\frac{3 \sigma}{\left(4 \beta^{2}-1\right) m^{2} L^{2}}\right)\left[1+\sigma \frac{3\left(4 \beta^{2}-3\right)}{\left(4 \beta^{2}-1\right) m^{2} L^{2}}\right]^{-1 / 2} .
\end{aligned}
$$

Using the Cardy formula, we also get the central charges,

$$
c=\eta \sigma \frac{L}{2 G} \frac{\sqrt{3\left(4 \beta^{2}-1\right)}}{\beta^{2}}\left(1+\frac{3 \sigma}{\left(4 \beta^{2}-1\right) m^{2} L^{2}}\right)\left[1+\sigma \frac{3\left(4 \beta^{2}-3\right)}{\left(4 \beta^{2}-1\right) m^{2} L^{2}}\right]^{-1 / 2},
$$

where $L$ and $\beta$ are given by the solution of Eqs (57) and (58).

These may be written as

$$
\begin{aligned}
S_{B H} & =\eta \sigma \frac{A_{H}}{4 G} \frac{4}{3 \sqrt{3-\beta^{2}}}=\eta \sigma \frac{A_{H}}{4 G} \frac{8}{3}\left(11+\frac{27 \sigma}{m^{2} L^{2}}\right)^{-1 / 2} \\
c & =\eta \sigma \frac{2 L}{G} \sqrt{\frac{\left(4 \beta^{2}-1\right)}{3\left(3-\beta^{2}\right)}} \frac{1}{\beta^{2}} .
\end{aligned}
$$

The central charge function formalism leads to central charge of the dual CFT as

$$
c=\eta \sigma \frac{L}{2 G} \sqrt{\operatorname{det} \mathcal{A}} \mathcal{B}=\frac{2 L}{3 G} \eta \frac{\left(\beta^{2}-4\right)}{\sqrt{3-\beta^{2}}},
$$

which is different from the above one.

\subsection{New Type Black Holes}

There are new type black hole solutions, which exist for a specific values of parameters in the Lagrangian in the $R^{3}$-NMG case 4 One can check these type black holes solutions exist only in the

\footnotetext{
${ }^{4}$ This is already pointed out in [18].
} 
$R^{3}$-NMG case. These black holes are shown to exist in the NMG case, but their properties are not investigated in detail even in the NMG case. (However, see [48] for some study of their properties and extension to the rotating new type black holes in the NMG case.) Its metric is given by

$$
d s^{2}=L^{2}\left[-\left(r^{2}+b r+c\right) d t^{2}+\frac{d r^{2}}{r^{2}+b r+c}+r^{2} d \phi^{2}\right]
$$

When $b$ is zero, this reduces the BTZ case of $r_{-}=0$.

To satisfy the EOM's, $l^{2}$ should satisfy the following equations

$$
l^{2}=\frac{\left(12 \sigma \xi m^{4}+\mu^{4}\right) L^{2}+6 \xi m^{2}}{m^{2}\left(8 \xi m^{2}+\mu^{4} L^{2}\right)},
$$

and $L^{2}$ is given by

$$
L^{2}=\frac{\sigma}{4 m^{2} \mu^{2}}\left(\mu^{2} \pm \sqrt{\mu^{4}+48 \sigma \xi m^{4}}\right) .
$$

Note that in the NMG limit (or $\xi \rightarrow 0$ ) these conditions become

$$
l^{2}=\frac{1}{m^{2}}, \quad L^{2}=\frac{1}{2 m^{2}}, \quad \sigma=1 .
$$

For the $b \neq 0$ case, the black hole horizon is at $r_{ \pm}=\left(-b \pm \sqrt{b^{2}-4 c}\right) / 2$ and the entropy and the temperature of the black holes are given by

$$
\begin{aligned}
S_{B H} & =\frac{\pi L}{2 G} \frac{\sqrt{b^{2}-4 c}}{3} \eta\left[2 \sigma+\frac{1}{2 m^{2} L^{2}}\right], \\
T_{H} & =\frac{\sqrt{b^{2}-4 c}}{4 \pi L}=\frac{r_{+}-r_{-}}{4 \pi L},
\end{aligned}
$$

where we have used the value of $L^{2}$ given in Eq. (64) to simplify the expression for the entropy. For the above static black hole solutions, one may use $A d S / C F T$ dictionary $S_{B H}=\frac{2 \pi^{2} L}{3} c T_{H}$ to obtain the central charge as

$$
c=\frac{L}{G} \eta\left[2 \sigma+\frac{1}{2 m^{2} L^{2}}\right]
$$

where $L^{2}$ is given in the Eq. (64). This result is consistent with [47] in the limit of $\xi=0$ with $\eta=\sigma=1$. One can see that this central charge is identical with (36) by noting that the chosen value of $L^{2}$ in (64) leads to

$$
\frac{\xi}{\mu^{4} L^{4}}=\frac{1}{3}\left(\sigma-\frac{1}{2 m^{2} L^{2}}\right)
$$

One way to define the mass of these black holes resorts to the $A d S / C F T$ dictionary through the relation $M=E_{L}+E_{R}$. In this case, this relation becomes $M=\frac{\pi^{2} L}{3} c T_{H}^{2}$, which leads to

$$
M=\frac{b^{2}-4 c}{48 G} \eta\left[2 \sigma+\frac{1}{2 m^{2} L^{2}}\right]
$$

This mass formula satisfies the simple form of the first law of black holes, $d M=T_{H} d S_{B H}$ and reduces to the correct value in the BTZ limit, $b=0$. However, it is unclear how to apply the 
first law of black holes in this case, since the nature of parameter $b$ is obscure. In fact, there is an attempt to realize the first law of black holes with the parameter $b$ and to understand its meaning as a new gravitational hair [48].

For new type black holes, the central charge function formalism leads to non-constant central charge. This fact is simply the indication that the curvature invariants of new type black holes are not constant. Therefore, we need some modification of the known central charge function formalism in this case. However, by recalling the fact that dual CFT resides in the boundary of bulk $\operatorname{AdS}$ space, it is very suggestive to define the central charge in this case as

$$
c=\left.\eta \sigma \frac{L}{2 G} \sqrt{\operatorname{det} \mathcal{A}} \mathcal{B}\right|_{r \rightarrow \infty},
$$

where $r$ is the radial coordinate and $r=\infty$ denotes the position of the boundary. Because the value obtained in this way is identical with the one by the Cardy formula, this formula is a natural generalization of the usual central charge function formula.

\section{Conclusion}

We have verified that BTZ, warped $A d S$ black holes and new type black holes are solutions in the extended new massive gravity theories, and investigated their properties in the view point of the AdS/CFT correspondence.

Firstly, we have presented various physical quantities, mass, angular momentum, Hawking temperature and entropy, of black holes in all the cases. Secondly, we have obtained central charges of hypothetical dual CFT using Cardy formula and central charge function formalism. Our results of entropy and central charges reduce to the known NMG cases in the NMG limit.

We have considered two version of extended NMG, $R^{3}-\mathrm{NMG}$ and BI-NMG, which seem to be related intimately. One may regard BI-NMG as a natural extension of $R^{3}$-NMG to the case of an infinite number of higher curvature terms. However, it needs to be clarified why the new type black holes exist only for the $R^{3}$-NMG case and whether the BI-NMG can be derived by the same argument for the extension of NMG to $R^{3}$-NMG.

We have obtained central charges of the dual CFTs by assuming the validity of Cardy formula, which is not self-transparent and may be thought as conjectural. Therefore, it will be interesting to obtain the central charges in other ways and verify our results, for example, by the approach in [25] 49] [51. Mass and angular momentum are also derived through the $A d S / C F T$ dictionary. It is required to obtain these quantities as conserved charges like in [44].

We have verified that the central charge function formalism leads to the same results with the Cardy formula for asymptotically $A d S$ black holes including new type black holes. For the new type black holes, we need a simple extension of central charge function formalism to match the results. However, in warped $A d S$ black holes, central charge function formalism, which is developed mainly for BTZ black holes, leads to different results from Cardy formula. It is very interesting to extend central charge function formalism to the warped $A d S$ case.

There are various direction to pursue in the future. First, though the gravitons are massive in the NMG case, it is not yet known that is the case for the extended NMG case. Therefore, it is 
necessary to analyze the linearized theory to see the nature of gravitons, and to see whether massive gravitons and positive mass black holes are compatible, which was not the case in the NMG or TMG case. It will also be interesting to study the possibility of string theory embedding or the supersymmetric extension of extended NMG.

Finally, new type black holes should be studied in more detail to see their meaning. They seem to have new type gravitational hair which is not yet understood completely. The fact that BI-NMG doesn't allow the new type black holes may also be addressed to see whether the existence of new type black holes is artifact or leads to some important lessons.

\section{Acknowlegements}

S.H.Y would like to thank the string theory group at Yonsei University, especially, Prof. Seungjoon Hyun for enlightening discussion. S.H.Y was supported by the National Research Foundation of Korea (NRF) grant funded by the Korea government (MEST) with the grant number 2009-0085995. This work of S.N and J.D.P was supported by a grant from the Kyung Hee University in 2009(KHU20100130). S.N was also supported by the National Research Foundation of Korea(NRF) grant funded by the Korean government(MEST)(No. 2009-0063068).

\section{APPENDIX}

In the NMG case, the reduced one-dimensional action derived from the action (11) is given by

$$
\begin{aligned}
S_{1}=\int & d \rho\left[A \zeta^{5}+B \zeta^{4} \zeta^{\prime}+C \zeta^{3} \zeta^{\prime 2}+D \zeta \zeta^{\prime 3}+E \zeta^{3}+F \zeta^{2} \zeta^{\prime}+G \zeta \zeta^{\prime 2}\right. \\
& \left.+H \zeta+J \zeta^{\prime}+\frac{2}{\ell^{2}} \zeta^{-1}\right] \\
=\int & d \rho\left[\left(A-\frac{1}{5} B^{\prime}\right) \zeta^{5}+C \zeta^{3} \zeta^{\prime 2}+D \zeta^{2} \zeta^{\prime 3}+\left(E-\frac{1}{3} F^{\prime}\right) \zeta^{3}+G \zeta \zeta^{\prime 2}\right. \\
& \left.+\left(H-J^{\prime}\right) \zeta+\frac{2}{\ell^{2}} \zeta^{-1}\right]
\end{aligned}
$$

where

$$
\begin{aligned}
A= & \frac{\xi}{12 \mu^{4}}\left\{-\frac{3}{8}\left(\vec{X}^{\prime 2}\right)^{3}-\frac{3}{2}\left(\vec{X}^{\prime 2}\right)^{2}\left(\vec{X} \cdot \vec{X}^{\prime \prime}\right)+6\left[\left(\vec{X}^{\prime 2}\right)+4\left(\vec{X} \cdot \vec{X}^{\prime \prime}\right)\right]\left(\vec{X} \wedge \vec{X}^{\prime \prime}\right)^{2}\right\} \\
B= & \frac{\xi}{12 \mu^{4}}\left\{-\frac{3}{2}\left(\vec{X}^{\prime 2}\right)^{2}\left(\vec{X} \cdot \vec{X}^{\prime}\right)+24\left(\vec{X} \cdot \vec{X}^{\prime}\right)\left(\vec{X} \wedge \vec{X}^{\prime \prime}\right)^{2}\right. \\
& \left.+12\left[\left(\vec{X}^{\prime 2}\right)+4\left(\vec{X} \cdot \vec{X}^{\prime \prime}\right)\right]\left(\vec{X} \wedge \vec{X}^{\prime}\right) \cdot\left(\vec{X} \wedge \vec{X}^{\prime \prime}\right)\right\} \\
E= & \frac{1}{m^{2}}\left\{\frac{1}{2}\left(\vec{X} \wedge \vec{X}^{\prime \prime}\right)^{2}-\frac{1}{4}\left(\vec{X}^{\prime 2}\right)\left(\vec{X} \cdot \vec{X}^{\prime \prime}\right)-\frac{3}{32}\left(\vec{X}^{\prime 2}\right)^{2}\right\}
\end{aligned}
$$




$$
\begin{aligned}
F & =\frac{1}{m^{2}}\left\{\left(\vec{X} \wedge \vec{X}^{\prime}\right) \cdot\left(\vec{X} \wedge \vec{X}^{\prime \prime}\right)-\frac{1}{4}\left(\vec{X}^{\prime 2}\right)\left(\vec{X} \cdot \vec{X}^{\prime}\right)\right\}, \\
H & =-\sigma\left\{\frac{3}{2}\left(\vec{X}^{\prime 2}\right)+2\left(\vec{X} \cdot \vec{X}^{\prime \prime}\right)\right\}, \\
J & =-2 \sigma\left(\vec{X} \cdot \vec{X}^{\prime}\right),
\end{aligned}
$$

and $(\vec{A} \wedge \vec{B}) \cdot(\vec{C} \wedge \vec{D})=-(\vec{A} \cdot \vec{C})(\vec{B} \cdot \vec{D})+(\vec{A} \cdot \vec{D})(\vec{B} \cdot \vec{C})$. Other terms are not needed to get equation of motion and Hamiltonian constraint.

The variation relative to $\vec{X}$ for the first form of the action (A.1) gives the equation of motion as following

$$
\delta A+\frac{1}{\zeta^{2}} \delta E+\frac{1}{\zeta^{4}} \delta H=0
$$

where

$$
\begin{aligned}
\delta A= & \frac{\xi}{12 \mu^{4}}\left\{\left(12\left(\overrightarrow{X^{\prime}}\right)+48\left(\vec{X} \cdot \vec{X}^{\prime \prime}\right)\right)\left[\vec{X} \wedge\left(\vec{X}^{\prime \prime \prime \prime} \wedge \vec{X}\right)\right]\right. \\
& +\left(24\left(\vec{X}^{\prime 2}\right)+96\left(\vec{X} \cdot \vec{X}^{\prime \prime}\right)\right)\left[\vec{X}^{\prime} \wedge\left(\vec{X}^{\prime \prime \prime} \wedge \vec{X}\right)+\vec{X} \wedge\left(\vec{X}^{\prime \prime \prime} \wedge \vec{X}^{\prime}\right)+\vec{X}^{\prime} \wedge\left(\vec{X}^{\prime \prime} \wedge \vec{X}^{\prime}\right)\right] \\
& +6\left(\vec{X}^{\prime 2}\right)\left[\vec{X}^{\prime \prime \prime} \wedge\left(\vec{X} \wedge \vec{X}^{\prime}\right)+\vec{X}^{\prime \prime} \wedge\left(\vec{X} \wedge \vec{X}^{\prime \prime}\right)\right]+12\left(\vec{X}^{\prime} \cdot \vec{X}^{\prime \prime}\right)\left[\vec{X}^{\prime \prime} \wedge\left(\vec{X} \wedge \vec{X}^{\prime}\right)\right] \\
& +\left(144\left(\vec{X}^{\prime} \cdot \vec{X}^{\prime \prime}\right)+96\left(\vec{X} \cdot \vec{X}^{\prime \prime \prime}\right)\right)\left[\vec{X} \wedge\left(\vec{X}^{\prime \prime \prime} \wedge \vec{X}\right)+\vec{X}^{\prime} \wedge\left(\vec{X}^{\prime \prime} \wedge \vec{X}\right)+\vec{X} \wedge\left(\vec{X}^{\prime \prime} \wedge \vec{X}^{\prime}\right)\right] \\
& +\left(72\left(\vec{X}^{\prime \prime 2}\right)+120\left(\vec{X}^{\prime} \cdot \vec{X}^{\prime \prime \prime}\right)+48\left(\vec{X} \cdot \vec{X}^{\prime \prime \prime \prime}\right)\right)\left[\vec{X} \wedge\left(\vec{X}^{\prime \prime} \wedge \vec{X}\right)\right] \\
& -\frac{3}{4}\left(\vec{X}^{\prime 2}\right)^{2} \vec{X}^{\prime \prime}+36\left(\vec{X} \wedge \vec{X}^{\prime \prime}\right)^{2} \vec{X}^{\prime \prime}+3\left(\vec{X}^{\prime 2}\right)\left(\vec{X}^{\prime} \cdot \vec{X}^{\prime \prime}\right) \vec{X}^{\prime} \\
& +72\left(\vec{X} \wedge \vec{X}^{\prime \prime}\right) \cdot\left(\vec{X}^{\prime} \wedge \vec{X}^{\prime \prime}\right) \vec{X}^{\prime}+72\left(\vec{X} \wedge \vec{X}^{\prime \prime}\right) \cdot\left(\vec{X} \wedge \vec{X}^{\prime \prime \prime}\right) \vec{X}^{\prime} \\
& +48\left(\vec{X}^{\prime} \wedge \vec{X}^{\prime \prime}\right){ }^{2} \vec{X}+48\left(\vec{X} \wedge \vec{X}^{\prime \prime \prime}\right) 2 \vec{X}+48\left(\vec{X} \wedge \vec{X}^{\prime \prime}\right) \cdot\left(\vec{X} \wedge \vec{X}^{\prime \prime \prime \prime}\right) \vec{X} \\
& \left.+96\left(\vec{X}^{\prime} \wedge \vec{X}^{\prime \prime}\right) \cdot\left(\vec{X} \wedge \vec{X}^{\prime \prime \prime}\right) \vec{X}+96\left(\vec{X} \wedge \vec{X}^{\prime \prime}\right) \cdot\left(\vec{X}^{\prime} \wedge \vec{X}^{\prime \prime \prime}\right) \vec{X}\right\} \cdot \delta \vec{X} \\
\delta E= & -\frac{1}{m^{2}}\left\{\vec{X} \wedge\left(\vec{X} \wedge \vec{X}^{\prime \prime \prime \prime}\right)+\frac{5}{2} \vec{X} \wedge\left(\vec{X}^{\prime} \wedge \vec{X}^{\prime \prime \prime}\right)+\frac{3}{2} \vec{X}^{\prime} \wedge\left(\vec{X} \wedge \vec{X}^{\prime \prime \prime}\right)\right. \\
& \left.+\frac{9}{4} \vec{X}^{\prime} \wedge\left(\vec{X}^{\prime} \wedge \vec{X}^{\prime \prime}\right)-\frac{1}{2} \vec{X}^{\prime \prime} \wedge\left(\vec{X} \wedge \vec{X}^{\prime \prime}\right)-\frac{1}{8}\left(\vec{X}^{\prime 2}\right) \vec{X}^{\prime \prime}\right\} \cdot \delta \vec{X} \\
\delta H= & -\sigma \vec{X}^{\prime \prime} \cdot \delta \vec{X} .
\end{aligned}
$$

The variation relative to $\zeta$ for the second form of the action (A.2) gives the Hamiltonian constraint

$$
\mathcal{H} \equiv\left(5 A-B^{\prime}\right)+\frac{1}{\zeta^{2}}\left(3 E-F^{\prime}\right)+\frac{1}{\zeta^{4}}\left(H-J^{\prime}\right)-\frac{2}{\ell^{2}} \frac{1}{\zeta^{6}}=0,
$$

where

$$
\begin{aligned}
B^{\prime}= & \frac{\xi}{12 \mu^{4}}\left\{-\frac{3}{2}\left(\vec{X}^{\prime 2}\right)^{3}-\frac{3}{2}\left(\vec{X}^{\prime 2}\right)^{2}\left(\vec{X} \cdot \vec{X}^{\prime \prime}\right)-6\left(\vec{X}^{\prime 2}\right)\left(\vec{X} \cdot \vec{X}^{\prime}\right)\left(\vec{X}^{\prime} \cdot \vec{X}^{\prime \prime}\right)\right. \\
& +36\left(\vec{X}^{\prime 2}\right)\left(\vec{X} \wedge \vec{X}^{\prime \prime}\right)^{2}+72\left(\vec{X} \cdot \vec{X}^{\prime \prime}\right)\left(\vec{X} \wedge \vec{X}^{\prime \prime}\right)^{2}+48\left(\vec{X} \cdot \vec{X}^{\prime}\right)\left(\vec{X} \wedge \vec{X}^{\prime \prime}\right) \cdot\left(\vec{X} \wedge \vec{X}^{\prime \prime}\right)^{\prime} \\
& +72\left(\vec{X}^{\prime} \cdot \vec{X}^{\prime \prime}\right)\left(\vec{X} \wedge \vec{X}^{\prime}\right) \cdot\left(\vec{X} \wedge \vec{X}^{\prime \prime}\right)+48\left(\vec{X} \cdot \vec{X}^{\prime \prime \prime}\right)\left(\vec{X} \wedge \vec{X}^{\prime}\right) \cdot\left(\vec{X} \wedge \vec{X}^{\prime \prime}\right) \\
& \left.+12\left(\vec{X}^{\prime 2}\right)\left(\vec{X} \wedge \vec{X}^{\prime}\right) \cdot\left(\vec{X} \wedge \vec{X}^{\prime \prime}\right)^{\prime}+48\left(\vec{X} \cdot \vec{X}^{\prime \prime}\right)\left(\vec{X} \wedge \vec{X}^{\prime}\right) \cdot\left(\vec{X} \wedge \vec{X}^{\prime \prime}\right)^{\prime}\right\}, \\
F^{\prime}= & \frac{1}{m^{2}}\left\{\left(\vec{X} \wedge \vec{X}^{\prime \prime}\right)^{2}+\left(\vec{X} \wedge \vec{X}^{\prime}\right) \cdot\left(\vec{X} \wedge \vec{X}^{\prime \prime \prime}\right)+\frac{3}{2}\left(\vec{X} \wedge \vec{X}^{\prime}\right) \cdot\left(\vec{X}^{\prime} \wedge \vec{X}^{\prime \prime}\right)\right.
\end{aligned}
$$




$$
\begin{aligned}
& \left.-\frac{1}{4}\left(\vec{X}^{\prime 2}\right)^{2}-\frac{3}{4}\left(\vec{X}^{\prime 2}\right)\left(\vec{X} \wedge \vec{X}^{\prime \prime}\right)\right\}, \\
J^{\prime}= & -2 \sigma\left\{\left(\vec{X}^{\prime 2}\right)+\left(\vec{X} \cdot \vec{X}^{\prime \prime}\right)\right\} .
\end{aligned}
$$

Then the Hamiltonian constraint can be represented as follows

$$
\begin{aligned}
\mathcal{H}= & \frac{\xi}{12 \mu^{4}}\left\{-\frac{3}{8}\left(\vec{X}^{\prime 2}\right)^{3}-6\left(\vec{X}^{\prime 2}\right)\left(\vec{X} \wedge \vec{X}^{\prime \prime}\right)^{2}-18\left(\vec{X}^{\prime 2}\right)\left(\vec{X} \wedge \vec{X}^{\prime}\right) \cdot\left(\vec{X}^{\prime} \wedge \vec{X}^{\prime \prime}\right)\right. \\
& -12\left(\vec{X}^{\prime 2}\right)\left(\vec{X} \wedge \vec{X}^{\prime}\right) \cdot\left(\vec{X} \wedge \vec{X}^{\prime \prime \prime}\right)-72\left(\vec{X}^{\prime} \cdot \vec{X}^{\prime \prime}\right)\left(\vec{X} \wedge \vec{X}^{\prime}\right) \cdot\left(\vec{X} \wedge \vec{X}^{\prime \prime}\right) \\
& +48\left(\vec{X} \cdot \vec{X}^{\prime \prime}\right)\left(\vec{X} \wedge \vec{X}^{\prime \prime}\right)^{2}-48\left(\vec{X} \cdot \vec{X}^{\prime \prime \prime}\right)\left(\vec{X} \wedge \vec{X}^{\prime}\right) \cdot\left(\vec{X} \wedge \vec{X}^{\prime \prime}\right) \\
& \left.-48\left(\vec{X} \cdot \vec{X}^{\prime}\right)\left(\vec{X} \wedge \vec{X}^{\prime \prime}\right) \cdot\left(\vec{X} \wedge \vec{X}^{\prime \prime}\right)^{\prime}-48\left(\vec{X} \cdot \vec{X}^{\prime \prime}\right)\left(\vec{X} \wedge \vec{X}^{\prime}\right) \cdot\left(\vec{X} \wedge \vec{X}^{\prime \prime}\right)^{\prime}\right\} \\
& +\frac{1}{\zeta^{2} m^{2}}\left\{\frac{1}{2}\left(\vec{X} \wedge \vec{X}^{\prime \prime}\right)^{2}-\frac{1}{32}\left(\vec{X}^{\prime 2}\right)^{2}-\left(\vec{X} \wedge \vec{X}^{\prime}\right) \cdot\left(\vec{X} \wedge \vec{X}^{\prime \prime \prime}\right)\right. \\
& \left.-\frac{3}{2}\left(\vec{X} \wedge \vec{X}^{\prime}\right) \cdot\left(\vec{X}^{\prime} \wedge \vec{X}^{\prime \prime}\right)\right\}+\frac{1}{\zeta^{4}} \frac{1}{2} \sigma\left(\vec{X}^{\prime 2}\right)-\frac{2}{\ell^{2} \zeta^{6}}=0 .
\end{aligned}
$$

In the Born-Infeld type case, the action is given by the form (3) and the determinant of matrix $\mathcal{A}^{\mu}{ }_{\nu}=\delta^{\mu}{ }_{\nu}+\frac{\sigma}{m^{2}} G^{\mu}{ }_{\nu}$ is represented as a form

$$
\operatorname{det} \mathcal{A}=1+\frac{\sigma}{m^{2}} F+\frac{1}{m^{4}} G+\frac{\sigma}{m^{6}} H
$$

where

$$
\begin{aligned}
F= & \zeta^{2}\left(\vec{X} \cdot \vec{X}^{\prime}\right)^{\prime}-\frac{1}{4} \zeta^{2}\left(\vec{X}^{\prime 2}\right)+\zeta \zeta^{\prime}\left(\vec{X} \cdot \vec{X}^{\prime}\right), \\
G= & \frac{1}{4} \zeta^{4}\left[\left(\vec{X} \cdot \vec{X}^{\prime}\right)^{\prime 2}-\left(\vec{L}^{\prime 2}\right)\right]-\frac{1}{16} \zeta^{4}\left(\vec{X}^{\prime 2}\right)^{2}+\frac{1}{2} \zeta^{3} \zeta^{\prime}\left[\left(\vec{X} \cdot \vec{X}^{\prime}\right)\left(\vec{X} \cdot \vec{X}^{\prime}\right)^{\prime}-\left(\vec{L} \cdot \vec{L}^{\prime}\right)\right] \\
& +\frac{1}{4} \zeta^{2} \zeta^{\prime 2}\left[\left(\vec{X} \cdot \vec{X}^{\prime}\right)^{2}-\left(\vec{L}^{2}\right)\right], \\
H= & \frac{1}{64} \zeta^{6}\left(\vec{X}^{\prime 2}\right)^{3}+\frac{1}{16} \zeta^{6}\left(\vec{X}^{\prime 2}\right)\left[\left(\vec{X} \cdot \vec{X}^{\prime}\right)^{\prime 2}-\left(\vec{X}^{\prime 2}\right)\left(\vec{X} \cdot \vec{X}^{\prime}\right)^{\prime}-\left(\vec{L}^{\prime 2}\right)\right] \\
& -\frac{1}{16} \zeta^{5} \zeta^{\prime}\left(\vec{X}^{\prime 2}\right)^{2}\left(\vec{X} \cdot \vec{X}^{\prime}\right)+\frac{1}{8} \zeta^{5} \zeta^{\prime}\left(\vec{X}^{\prime 2}\right)\left[\left(\vec{X} \cdot \vec{X}^{\prime}\right)\left(\vec{X} \cdot \vec{X}^{\prime}\right)^{\prime}-\left(\vec{L} \cdot \vec{L}^{\prime}\right)\right] \\
& +\frac{1}{16} \zeta^{4} \zeta^{\prime 2}\left(\vec{X}^{\prime 2}\right)\left[\left(\vec{X} \cdot \vec{X}^{\prime}\right)^{2}-\left(\vec{L}^{2}\right)\right] .
\end{aligned}
$$

The variation of the BI type action relative to $\vec{X}$ for equation of motion is given by

$$
\begin{aligned}
\delta \mathcal{S} \simeq & \int d \rho \frac{1}{8 \zeta}(\operatorname{det} \mathcal{A})^{-5 / 2}\left[4(\operatorname{det} \mathcal{A})^{2}\left(\vec{S}^{\prime \prime}-\vec{Q}^{\prime}+\vec{P}\right)-4(\operatorname{det} \mathcal{A})\left(\operatorname{det} \mathcal{A}^{\prime}\right) \vec{S}^{\prime}\right. \\
& \left.-2(\operatorname{det} \mathcal{A})\left(\operatorname{det} \mathcal{A}^{\prime \prime}\right) \vec{S}+3\left(\operatorname{det} \mathcal{A}^{\prime}\right)^{2} \vec{S}+2(\operatorname{det} \mathcal{A})\left(\operatorname{det} \mathcal{A}^{\prime}\right) \vec{Q}\right] \cdot \delta \vec{X}
\end{aligned}
$$

From this variation of action, we can obtain the equation of motion

$$
\begin{aligned}
4(\operatorname{det} \mathcal{A})^{2}\left(\vec{S}^{\prime \prime}-\vec{Q}^{\prime}+\vec{P}\right)- & 4(\operatorname{det} \mathcal{A})\left(\operatorname{det} \mathcal{A}^{\prime}\right) \vec{S}^{\prime}-2(\operatorname{det} \mathcal{A})\left(\operatorname{det} \mathcal{A}^{\prime \prime}\right) \vec{S} \\
& +3\left(\operatorname{det} \mathcal{A}^{\prime}\right)^{2} \vec{S}+2(\operatorname{det} \mathcal{A})\left(\operatorname{det} \mathcal{A}^{\prime}\right) \vec{Q}=0
\end{aligned}
$$


where

$$
\begin{aligned}
\vec{P}= & \sigma \frac{\zeta^{2}}{m^{2}} \vec{X}^{\prime \prime}+\frac{\zeta^{4}}{m^{4}}\left[\frac{1}{2}\left(\vec{X}^{\prime 2}\right) \vec{X}^{\prime \prime}+\frac{1}{2}\left(\vec{X} \cdot \vec{X}^{\prime}\right) \vec{X}^{\prime \prime}+\frac{1}{2}\left(\vec{X}^{\prime \prime 2}\right) \vec{X}\right. \\
& \left.-\frac{1}{2}\left(\vec{X} \cdot \vec{X}^{\prime \prime}\right) \vec{X}^{\prime \prime}\right]+\sigma \frac{\zeta^{6}}{m^{6}}\left[\frac{1}{16}\left(\vec{X}^{\prime 2}\right)^{2} \vec{X}^{\prime \prime}+\frac{1}{8}\left(\vec{X}^{\prime 2}\right)\left(\vec{X} \cdot \vec{X}^{\prime \prime}\right) \vec{X}^{\prime \prime}\right. \\
& \left.+\frac{1}{8}\left(\vec{X}^{\prime 2}\right)\left(\vec{X}^{\prime \prime 2}\right) \vec{X}-\frac{1}{8}\left(\vec{X}^{\prime 2}\right)\left(\vec{X} \cdot \vec{X}^{\prime \prime}\right) \vec{X}^{\prime \prime}\right] \\
\vec{Q}= & \sigma \frac{\zeta^{2}}{m^{2}} \frac{3}{2} \vec{X}^{\prime}+\frac{\zeta^{4}}{m^{4}}\left[\frac{3}{4}\left(\vec{X}^{\prime 2}\right) \vec{X}^{\prime}+\left(\vec{X} \cdot \vec{X}^{\prime \prime}\right) \vec{X}^{\prime}\right]+\sigma \frac{\zeta^{6}}{m^{6}}\left[\frac{3}{32}\left(\vec{X}^{\prime 2}\right)^{2} \vec{X}^{\prime}\right. \\
& \left.+\frac{1}{4}\left(\vec{X}^{\prime 2}\right)\left(\vec{X} \cdot \vec{X}^{\prime \prime}\right) \vec{X}^{\prime}+\frac{1}{8}\left(\vec{X} \cdot \vec{X}^{\prime \prime}\right)^{2} \vec{X}^{\prime}-\frac{1}{8}\left(\vec{X} \wedge \vec{X}^{\prime \prime}\right)^{2} \vec{X}^{\prime}\right], \\
\vec{S}= & \sigma \frac{\zeta^{2}}{m^{2}} \vec{X}+\frac{\zeta^{4}}{m^{4}}\left[\frac{1}{2}\left(\vec{X}^{\prime 2}\right) \vec{X}+\frac{1}{2}\left(\vec{X}^{2}\right) \vec{X}^{\prime \prime}\right]+\sigma \frac{\zeta^{6}}{m^{6}}\left[\frac{1}{16}\left(\vec{X}^{\prime 2}\right)^{2} \vec{X}\right. \\
& \left.+\frac{1}{8}\left(\vec{X}^{\prime 2}\right)\left(\vec{X} \cdot \vec{X}^{\prime \prime}\right) \vec{X}+\frac{1}{8}\left(\vec{X}^{2}\right)\left(\vec{X}^{\prime 2}\right) \vec{X}^{\prime \prime}-\frac{1}{8}\left(\vec{X}^{\prime 2}\right)\left(\vec{X} \cdot \vec{X}^{\prime \prime}\right) \vec{X}\right]
\end{aligned}
$$

with $\zeta=$ const.

The variation of the action with $\zeta$ is given by

$$
\begin{aligned}
\delta_{\zeta} \mathcal{S} \sim & \int d \rho \frac{1}{2 \zeta^{2}}(\operatorname{det} \mathcal{A})^{-1 / 2}\left\{\mathcal{M}+\frac{1}{2} \frac{\left(\operatorname{det} \mathcal{A}^{\prime}\right)}{\operatorname{det} \mathcal{A}} \mathcal{N}-\mathcal{N}^{\prime}\right. \\
& \left.-2\left[(\operatorname{det} \mathcal{A})-(\operatorname{det} \mathcal{A})^{1 / 2}\left(1+\frac{1}{2 m^{2} \ell^{2}}\right)\right]\right\} \delta \zeta
\end{aligned}
$$

From the above variation of the action, we can get the Hamiltonian constraint

$$
\frac{1}{2} \frac{\left(\operatorname{det} \mathcal{A}^{\prime}\right)}{\operatorname{det} \mathcal{A}} \mathcal{N}+\mathcal{M}-\mathcal{N}^{\prime}-2(\operatorname{det} \mathcal{A})+2(\operatorname{det} \mathcal{A})^{1 / 2}\left(1+\frac{1}{2 m^{2} \ell^{2}}\right)=0
$$

where

$$
\begin{aligned}
\mathcal{M}= & \sigma \frac{\zeta^{2}}{m^{2}}\left[2\left(\vec{X} \cdot \vec{X}^{\prime}\right)^{\prime}-\frac{1}{2}\left(\vec{X}^{\prime 2}\right)\right]+\frac{\zeta^{4}}{m^{4}}\left[\left(\vec{X} \cdot \vec{X}^{\prime}\right)^{\prime 2}-\frac{1}{4}\left(\vec{X}^{\prime 2}\right)^{2}-\left(\vec{L}^{\prime 2}\right)\right] \\
& +\sigma \frac{\zeta^{6}}{m^{6}}\left[\frac{3}{32}\left(\vec{X}^{\prime 2}\right)^{3}+\frac{3}{8}\left(\vec{X}^{\prime 2}\right)\left(\vec{X} \cdot \vec{X}^{\prime}\right)^{\prime 2}-\frac{3}{8}\left(\vec{X}^{\prime 2}\right)^{2}\left(\vec{X} \cdot \vec{X}^{\prime}\right)^{\prime}-\frac{3}{8}\left(\vec{X}^{\prime 2}\right)\left(\vec{L}^{\prime 2}\right)\right], \\
\mathcal{N}= & \sigma \frac{\zeta^{2}}{m^{2}}\left(\vec{X} \cdot \vec{X}^{\prime}\right)+\frac{\zeta^{4}}{m^{4}}\left[\frac{1}{2}\left(\vec{X} \cdot \vec{X}^{\prime}\right)\left(\vec{X} \cdot \vec{X}^{\prime}\right)^{\prime}-\frac{1}{2}\left(\vec{L} \cdot \vec{L}^{\prime}\right)\right] \\
& +\sigma \frac{\zeta^{6}}{m^{6}}\left[-\frac{1}{16}\left(\vec{X}^{\prime 2}\right)^{2}\left(\vec{X} \cdot \vec{X}^{\prime}\right)+\frac{1}{8}\left(\vec{X}^{\prime 2}\right)\left(\vec{X} \cdot \vec{X}^{\prime}\right)\left(\vec{X} \cdot \vec{X}^{\prime}\right)^{\prime}-\frac{1}{8}\left(\overrightarrow{X^{\prime 2}}\right)\left(\vec{L} \cdot \vec{L}^{\prime}\right)\right]
\end{aligned}
$$

with $\zeta=$ const. 


\section{References}

[1] E. A. Bergshoeff, O. Hohm and P. K. Townsend, "Massive Gravity in Three Dimensions," Phys. Rev. Lett. 102 (2009) 201301 arXiv:0901.1766 [hep-th]].

[2] S. Deser, R. Jackiw and S. Templeton, "Topologically massive gauge theories," Annals Phys. 140, 372 (1982) [Erratum-ibid. 185, 406.1988 APNYA,185,406.1988 APNYA,281,409 (1988 APNYA,185,406.1988 APNYA,2].

[3] R. Jackiw, S. Templeton and S. Deser, "Three-Dimensional Massive Gauge Theories," Phys. Rev. Lett. 48 (1982) 975.

[4] K. Hotta, Y. Hyakutake, T. Kubota and H. Tanida, Brown-Henneaux's Canonical Approach to Topologically Massive Gravity," JHEP 0807 (2008) 066 [arXiv:0805.2005 [hep-th]].

[5] I. Vuorio, "Topologically Massive Planar Universe," Phys. Lett. B 163 (1985) 91.

[6] R. Percacci, P. Sodano and I. Vuorio, "Topologically Massive Planar Universes With Constant Twist," Annals Phys. 176 (1987) 344.

[7] Y. Nutku, "Exact solutions of topologically massive gravity with a cosmological constant," Class. Quant. Grav. 10 (1993) 2657.

[8] M. Gürses, Class. Quant. Grav. 11 (1994) 2585.

[9] A. Bouchareb and G. Clément, "Black hole mass and angular momentum in topologically massive gravity," Class. Quant. Grav. 24 (2007) 5581 [arXiv:0706.0263 [gr-qc]].

[10] E. A. Bergshoeff, O. Hohm and P. K. Townsend, "More on Massive 3D Gravity," Phys. Rev. D 79 (2009) 124042 [arXiv:0905.1259 [hep-th]].

[11] G. Clément, "Warped $A d S_{3}$ black holes in new massive gravity," Class. Quant. Grav. 26 (2009) 105015 arXiv:0902.4634 [hep-th]].

[12] Y. Liu and Y. Sun, "Note on New Massive Gravity in AdS(3)," JHEP 0904 (2009) 106 arXiv:0903.0536 [hep-th]].

[13] Y. Liu and Y. Sun, "Consistent Boundary Conditions for New Massive Gravity in $A d S_{3}$," JHEP 0905 (2009) 039 arXiv:0903.2933 [hep-th]].

[14] E. Ayon-Beato, A. Garbarz, G. Giribet and Mokhtar Hassaine, "Lifshitz Black Hole in Three Dimensions," Phys. Rev. D 80 (2009) 104029 [arXiv:0909.1347 [hep-th]].

[15] E. Ayon-Beato, G. Giribet and M. Hassaine, "Bending AdS Waves with New Massive Gravity," JHEP 0905 (2009) 029 arXiv:0904.0668 [hep-th]].

[16] E. Bergshoeff, O. Hohm, P. Townsend, "On Higher Derivatives in 3D Gravity and Higher Spin Gauge Theories," Annals Phys. 325 (2010) 1118 [arXiv:0911.3061 [hep-th]].

[17] R. Andringa, E. Bergshoeff, M. de Roo, O. Hohm, E. Sezgin, P. Townsend, "Massive 3D Supergravity," Class. Quant. Grav. 27 (2010) 025010 [arXiv:0907.4658 [hep-th]]

[18] A. Sinha, "On the new massive gravity and AdS/CFT," arXiv:1003.0683 [hep-th]]. 
[19] I. Gullu, T. C. Sisman and B. Tekin, "Born-Infeld extension of new massive gravity," arXiv:1003.3935 [hep-th]].

[20] J. Maldacena, "The Large N limit of superconformal field theories and supergravity," Adv. Theor. Math. Phys. 2 (1998) 231, Int. J. Theor. Phys. 38(1999) 1113 arXiv:hep-th/9711200.

[21] E. Witten, "Anti-de Sitter space and holography, Adv. Theor. Math. Phys. 2(1998) 253 arXiv:hep-th/9802150.

[22] E. Witten, "Anti-de Sitter space, thermal phase transition, and confinement in gauge theories," Adv. Theor. Math. Phys. 2 (1998) 505 [arXiv:hep-th/9803131].

[23] J. L. Cardy, "Operator Content of Two-Dimensional Conformally Invriant Theories," Nucl. Phys. B 270 (1986) 186.

[24] S. Carlip, "What we don't know about BTZ black hole entropy," Class. Quant. Grav. 15 (1998) 3609 [arXiv:hep-th/9806026].

[25] J. D. Brown, M. Henneaux, "Central Charges in the Canonical Realization of Asymptotic Symmetries: An Example from Three-Dimensional Gravity," Commun. Math. Phys. 104 (1986) 207.

[26] M. Henningson and K. Skenderis, "The holographic Weyl anomaly," JHEP 9807 (1998) 023 arXiv:hep-th/9806087.

[27] V. Balasubramanian and P. Kraus, "A stress tensor for anti-de Sitter gravity," Commun. Math. Phys. 208 (1999) 413 arXiv:hep-th/9902121.

[28] S. de Haro, S. N. Solodukhin and K. Skenderis, "Holographic reconstruction of spacetime and renormalization in the AdS/CFT correspondence," Commun. Math. Phys. 217 (2001) 595 arXiv:hep-th/0002230.

[29] P. Kraus and F. Larsen, "Microscopic Black Hole Entropy in Theories with Higher Derivatives," JHEP 0509 (2005) 034 arXiv:hep-th/0506176].

[30] E. Bergshoeff, O. Hohm and P. Townsend, "On massive gravitons in 2+1 dimensions," arXiv:0912.2944 [hep-th]].

[31] G. Clément, "Classical solutions in three-dimensional cosmological gravity," Phys. Rev. D 49 (1994) 5131.

[32] J. Lee and R. Wald, "Local symmetries and constraints," J. Math. Phys. 31 (1990) 725.

[33] R. Wald, "On identically closed forms locally constructed from a field," J. Math. Phys. 31 (1990) 2378.

[34] R. M. Wald, "Black hole entropy is the Noether charge," Phys. Rev. D 48 (1993) 3427 arXiv:gr-qc/9307038.

[35] T. Jacobson, G. Kang and R. C. Myers, "On Black Hole Entropy," Phys. Rev. D 49 (1994) 6587 arXiv:gr-qc/9312023. 
[36] T. Jacobson, G. Kang and R. Myers, "Black hole entropy in higher curvature gravity," arXiv:gr-qc/9502009].

[37] H. Saida and J. Soda, "Statistical entropy of BTZ black hole in higher curvature gravity," Phys. Lett. B 471 (2000) 358 arXiv:gr-qc/9909061.

[38] G. Clement, "Particle - like solutions to topologically massive gravity," Class. Quant. Grav. 11 (1994) L115 arXiv:gr-qc/9404004.

[39] K. A. Moussa, G. Clément and C. Leygnac, "The Black holes of topologically massive gravity," Class. Quant. Grav. 20 (2003) L277 arXiv:gr-qc/0303042.

[40] K. A. Moussa, G. Clement, H. Guennoune and C. Leygnac, Three-dimensional Chern-Simons black holes," Phys. Rev. D 78 (2008) 064065 arXiv:0807.4241 [gr-qc]].

[41] M. Banados, C. Teitelboim and J. Zanelli, The Black hole in three-dimensional space-time," Phys. Rev. Lett. 69 (1992) 1849 arXiv:hep-th/9204099].

[42] M. Banados, M. Henneaux, C. Teitelboim and J. Zanelli, "Geometry of the $(2+1)$ black hole," Phys. Rev. D 48 (1993) 1506 arXiv:gr-qc/9302012.

[43] J. Maldacena and A. Strominger, "AdS(3) black holes and a stringy exclusion principle." JHEP 9812 (1998) 005 arXiv:hep-th/9804085.

[44] S. Deser and B. Tekin, "New Energy Definition for Higher Curvature Gravities," Phys. Rev. D 75 (2007) 084032 arXiv:gr-qc/0701140.

[45] D. Anninos, W. Li, M. Padi, W. Song and A. Strominger, "Warped $A d S_{3}$ Black Holes," JHEP 0903 (2009) 130 [arXiv:0807.3040 [hep-th]].

[46] W. Kim and E. J. Son, "Central Charges in 2d Reduced Cosmological Massive Gravity," Phys. Lett. B 678, 107 (2009) arXiv:0904.4538 [hep-th]].

[47] J. Oliva, D. Tempo and R. Troncoso, "Three-dimensional black holes, gravitational solitons, kinks and wormholes for BHT masive gravity," JHEP 0907 (2009) 011 arXiv:0905.1545 [hepth]].

[48] G. Giribet, J. Oliva, D. Tempo and R. Troncoso, "Microscopic entropy of the three-dimensional rotating black hole of BHT massive gravity," Phys. Rev. D 80 (2009) 124046 [arXiv:0909.2564 [hep-th]].

[49] G. Compere and S. Detournay, "Semi-classical central charge in topologically massive gravity," Class. Quant. Grav. 26 (2009) 012001, Erratum-ibid. 26 (2009) 139801 [arXiv:0808.1911] [hepth]].

[50] G. Compere and S. Detournay, "Boundary conditions for spacelike and timelike warped $A d S_{3}$ spaces in topologically massive gravity," JHEP 0908 (2009) 092 [arXiv:0906.1243 [hep-th]].

[51] M. Blagojevic, B. Cvetkovic, "Asymptotic structure of topologically massive gravity in spacelike stretched AdS sector," JHEP 0909 (2009) 006 [arXiv:0907.0950 [gr-qc]]. 